\title{
ON DYNAMIC DECOUPLING AND DYNAMIC PATH CONTROLLABILITY IN ECONOMIC SYSTEMS
}

\author{
Henk NIJMEIJER* \\ University of Twente, 7500 AE Enschede, The Netherlands \\ Received September 1987, final version received May 1988
}

In this paper the dynamic decouplability and dynamic path controllability of nonlinear discretetime economic systems in state space form are discussed. Based on the observation that both properties are equivalent, a (theoretical) efficient way of target path controllability is proposed. This is illustrated for a fairly general example of a closed economy.

\section{Introduction}

In the theory of economic policy making a standard question deals with the ability of guiding a given set of target variables along arbitrarily chosen time paths by a suitable selection of the policy instruments at each time instant. The rationale of having a positive answer to this question of dynamic path controllability or perfect controllability is of course that in such a situation the policymaker can steer the target variables along desired paths. This could be interpreted as a dynamic generalization of the well-known Tinbergen concept of static controllability. Dynamic path controllability has been studied in the economics literature by various authors, e.g., Aoki $(1974,1975,1976)$, Buiter (1979), Aoki and Canzoneri (1979), Preston and Pagan (1982), Wohltmann (1984, 1985b), Wohltmann and Krömer $(1983,1984)$, and others. There also exists a large engineering literature on dynamic path controllability - in that context often referred to as right-invertibility or functional reproducibility - see, e.g., Brockett and Mesarovic (1965), Sain and Massey (1969), Wolovich (1974), and others. In all forementioned references one has to assume that the underlying (economic) model is a linear one in state space form. The linearity requirement is clearly usually not met. To avoid nonlinearities in the model a common approach is that one linearizes the economic model around a fixed equilibrium point and then one applies the linear theory on dynamic path

\footnotetext{
*The author is grateful to an anonymous referee for valuable comments on an earlier draft of this paper.
} 
controllability. To what extent this method may be successfully applied of course fully depends heavily on the ignored higher-order terms in the linearization.

Another approach for handling dynamic path controllability in a dynamic system is based on decoupling. In a set-up for decoupling one requires that a given instrument variable only influences one particular target variable and none of the other target variables and each target variable is affected by only one instrument variable. Usually a situation with this noninteracting property is not met and one may ask for automatic policy rules which do achieve a decoupled system. Notice that a decoupled system automatically has the dynamic path controllability property. Each instrument variable can be used to control its corresponding target variable time path. In this context one needs a good understanding of automatic policy rules or fcedback control loops as this is called in engineering. The concept of decoupling has been treated in the economic literature in Aoki $(1974,1976)$ and Aoki and Canzoneri (1979) and in engineering in Falb and Wolovich (1967), Wang (1970), and Sain and Massey (1969), and many others. Again all these references are concerned with linear dynamic models, although in engineering in the last decade also the decoupling problem for dynamic continuous-time nonlinear systems has received much attention; see, e.g., Sinha (1977), Claude (1983), Isidori (1985), Descusse and Moog (1985), Nijmeijer (1986), and Nijmeijer and Respondek (1986). Very recently a similar theory for discrete-time nonlinear models has been developed in Nijmeijer (1987). The last reference forms the starting point of the present note.

As stated, the connection between target path controllability and decouplability of a system is, at least for linear systems, not completely new. In particular, in an economic context Aoki and Canzoneri (1979) give an explicit connection between target path controllability and static state feedback decoupling (see section 2 for precise definitions and results), though unfortunately, as will be shown by means of an example, their results are not completely correct at this point. In the present note we take the most general and weakest notion of decoupling: the automatic policy rulcs we allow for may contain some dynamic elements - to be designed by the policy maker. The classical result on static decoupling [see, e.g., Falb and Wolovich (1967)] is therefore included in the results given here.

Throughout the paper we consider discrete-time economic models. On a conceptual level this simplifies things a bit but both for linear and nonlinear systems one can extend in principle the main results also to continuous-time models; see, e.g., Wolovich (1974) and Nijmeijer and Respondek (1986). However, already for linear continuous-time systems one further complication arises in specifying the admissible function spaces for the target and instrument variables, see also Wohltmann $(1985 \mathrm{a}, \mathrm{b})$ and Albrecht, Grasse and Wax (1981). This is due to the fact that the desired instrument variable which is 
needed for the system to follow a prescribed target path appears as a function of the time derivatives of the desired output function; cf. Lovass-Nagy, Miller and Powers $(1976,1978)$. In fact, as their techniques clearly illustrate, the desired instrument variable is obtained via the construction of an 'inverse' of the original model. The frequency domain approach of Maciejowski and Vines (1984) is essentially based on the same idea of producing a given target path via the construction of an inverse in the frequency domain. As in this last reference an 'inverse' model approach for discrete-time linear systems will not encounter the forementioned difficulties and could also be used in the study of target path controllability. Note that the idea of using an 'inverse' model is also given for continuous time nonlinear systems in Albrecht, Grasse and Wax (1981).

The purpose of this note is to present a theory for dynamic path controllability of nonlinear discrete-time dynamical systems and to discuss the connection between dynamic path controllability and decouplability of such systems. In section 2 the notion of dynamic decoupling is introduced and the equivalence between dynamic path controllability and dynamic decoupling is discussed. In section 3 for a particular example a detailed study of an algorithm for verifying dynamic decoupling - and thus dynamic path controllability - is given. The obtained results agree with the ones obtained in Wohltmann and Krömer (1984) where a linearized version of the same example is discussed.

\section{Decouplability and dynamic path controllability}

It is convenient to express the model under consideration in state space form. This allows for a uniform theoretical treatment. A state space representation of a discrete-time economic model is generally given by

$$
\begin{aligned}
& q(k+1)=f(q(k), u(k), w(k)), \\
& y(k)=h(q(k), u(k), w(k)),
\end{aligned}
$$

where $q$ is an $n$-dimensional state vector, $u$ is an $m$-dimensional instrument vector consisting of the policy variables that can be freely assessed, $w$ is an $r$-dimensional vector of (known) exogenous variables (remaining policy variables, etc.), and $y$ is a $p$-dimensional vector of target variables that the policy maker wants to control. The state transition function $f$ and the output function $h$ are supposed to be smooth, say at least $n$ times differentiable. When necessary we denote the components of a vector by using lower indices, e.g., $\quad q(k)=\left(q_{1}(k), \ldots, q_{n}(k)\right)^{\top}, \quad h(q, u, w)=\left(h_{1}(q, u, w), \ldots, h_{p}(q, u, w)\right)^{\top}$. 
The simplest class of models of the type (1) consists of the linear ones

$$
\begin{aligned}
& q(k+1)=A q(k)+B u(k)+E w(k), \\
& y(k)=C q(k)+D u(k)+I w(k),
\end{aligned}
$$

where $A, B, E, C, D$ and $I$ are matrices of appropriate size. To keep the exposition as clear as possible we will assume throughout that the number of instrument variables equals the number of target variables, i.e.,

$$
m=p
$$

although the whole theory can be developed also when $m>p$. In the sequel it will be shown that this dynamic generalization of the Tinbergen condition $m \geq p$ is necessary in the decoupling and dynamic path controllability; cf. Wohltmann and Krömer (1984). The system (1) is called decoupled if - possibly after relabeling the instrument variables - the $i$ th instrument variable $u_{i}(\cdot)$ only affects the $i$ th target variable $y_{i}(\cdot)$ and none of the other target variables $y_{j}(\cdot), j \neq i, i=1, \ldots, m$. For the linear system (2) this is equivalent to the condition that the $z$-transform or discrete Laplace transform of instrument variables to target variables of (2),

$$
H(z)=C\left(z I_{n}-A\right)^{-1} B+D \quad\left[I_{n}:(n, n) \text { identity matrix }\right]
$$

is an $(m, m)$ diagonal matrix.

Whenever the system (1) or (2) is not yet decoupled one may ask whether this interdependence can be undone or offset through the use of appropriate control rules. The simplest way of trying to do so is by introducing a new vector of instrument variables $v=\left(v_{1}, \ldots, v_{m}\right)^{\top}$ via

$$
u(k)=\alpha(q(k), v(k))
$$

for the system (1), or for the linear system (2) the linear control law

$$
u(k)=F q(x)+G v(k),
$$

and the question then is when does there exist such a policy that decouples the system. See for the linear problem also Aoki (1974) and Aoki and Canzoneri (1979), and Nijmeijer (1987) for the nonlinear problem. We will refer to this as the static decoupling problem. Let us briefly discuss the solvability of the linear problem. Consider the system (2) and assume for the 
moment $w(\cdot) \equiv 0$, i.e.,

$$
\begin{aligned}
& q(k+1)=A q(k)+B u(k), \\
& y(k)=C q(k)+D u(k) .
\end{aligned}
$$

Consider the $i$ th output $y_{i}(k)=C_{i} q(k)+D_{i} u(k)$. Either $D_{i}-$ the $i$ th row of $D$ - is zero or nonzero. Whenever $D_{i} \neq 0$, we observe that $y_{i}(k)$ explicitly depends upon $u(k)$ and, when $D_{i}=0$, then $y_{i}(k)$ is not explicitly depending on $u(k)$. When $D_{i}=0$, we see that $y_{i}(k+1)=C_{i} A q(k)+C_{i} B u(k)$, and this explicitly depends on $u(k)$ iff $C_{i} B \neq 0$. In this manner we can introduce an integer $\rho_{i}$ as being the minimal number for which $y_{i}\left(k+\rho_{i}\right)$ is explicitly depending on $u(k)$, so $\rho_{i}=0$ if $D_{i} \neq 0$ and if $D_{i}=0 \rho_{i}$ is defined as the minimal number $l$ for which $C_{i} A^{l-1} B \neq 0$. Having defined the characteristic numbers $\rho_{1}, \ldots, \rho_{m}$ we form the $(m, m)$ matrix $M$, called the decoupling matrix, of which the $i$ th row is given as $D_{i}$ when $\rho_{i}=0$ and $C_{i} A^{\rho_{i}-1} B$ when $\rho_{i}>0$. So we have

$$
\left(\begin{array}{c}
y_{1}\left(k+\rho_{1}\right) \\
\vdots \\
y_{m}\left(k+\rho_{m}\right)
\end{array}\right)=\left(\begin{array}{c}
C_{1} A^{\rho_{1}} \\
\vdots \\
C_{m} A^{\rho_{m}}
\end{array}\right) q(k)+M u(k)
$$

The following theorem gives a complete solution to the static decoupling problem.

Theorem 1. The system (7) is static decouplable via the control law (6) if and only if the matrix $M$ is nonsingular.

Proof. See Falb and Wolovich (1967).

We remark that some of the sufficient conditions formulated in Aoki and Canzoneri (1979) are special cases of the here given necessary and sufficient conditions. An easy way to determine possible $F$ and $G$ in (6), which achieve decoupling, goes as follows. Define the right-hand side of (8) to be equal to $v(k)=\left(v_{1}(k), \ldots, v_{m}(k)\right)^{\top}$ and solve this equation for $u(k)$ as a function of $q(k)$ and $v(k)$ :

$$
u(k)=-M^{-1}\left(\begin{array}{c}
C_{1} A^{\rho_{1}} \\
\vdots \\
C_{m} A^{\rho_{m}}
\end{array}\right) q(k)+M^{-1} v(k)
$$


which is precisely of the desired form (6) if we set

$$
F=-M^{-1}\left(C_{1} A^{\rho_{1}}, \ldots, C_{m} A^{\rho_{m}}\right)^{\top} \text { and } G=M^{-1} \text {. }
$$

Note that the computations involved can be rather straightforward implemented on a microcomputer. Applying (9) to (7) yields

$$
\begin{gathered}
y_{1}\left(k+\rho_{1}\right)=v_{1}(k), \\
\vdots \\
y_{m}\left(k+\rho_{m}\right)=v_{m}(k) .
\end{gathered}
$$

Applying the same control law (9) to the system (7) with exogenous variables gives

$$
\begin{aligned}
y_{1}\left(k+\rho_{1}\right)= & v_{1}(k)+I_{1} w\left(k+\rho_{1}\right)+I_{1}(A+B F) w\left(k+\rho_{1}-1\right) \\
& +\cdots+I_{1}(A+B F)^{\rho_{1}} w(k), \\
\vdots & \\
y_{m}\left(k+\rho_{m}\right)= & v_{m}(k)+I_{m} w\left(k+\rho_{m}\right)+I_{m}(A+B F) w\left(k+\rho_{m}-1\right) \\
& +\cdots+I_{m}(A+B F)^{\rho_{m}} w(k),
\end{aligned}
$$

which, of course, is still decoupled. Note that by a suitable choice of $v_{i}(\cdot)$ one can compensate the exogenous effect on each of the target variables. So, if we have full freedom in choosing the new instrument variables $\left(v_{1}, \ldots, v_{m}\right)$, a trend may be eliminated.

For the nonlinear model (1) with constant exogenous instruments a similar approach yields an explicit way of computing a control strategy (5) which decouples the nonlinear system into the form (10) although for each time instance the controls may be bounded to some region; sce Nijmeijer (1987) for a complete treatment. In the next section we will discuss this in detail for a specific example. So far we have only discussed decoupling via policies of the form (5) or, for a linear system, of the form (6). Of course one could allow for far more general control policies. In the study of decoupling the most appropriate general policy control rules are defined as follows. Introduce another state space model (to be designed by the policy maker)

$$
\begin{aligned}
& x(k+1)=\phi(x(k), \hat{u}(k)), \\
& u(k)=\psi(x(k), \hat{u}(k)),
\end{aligned}
$$

where $x$ is an $\nu$-dimensional state vector, $\hat{u}$ is an $m$-dimensional new instru- 
ment vector, and $\nu$ and the functions $\phi$ and $\psi$ are still to be determined. Eqs. (1) and (12) together define a new model with as states $(q(\cdot), x(\cdot))^{\top}$, instrument variables $\hat{u}(\cdot)$, and target variables as before $y(\cdot)$, so

$$
\begin{aligned}
& q(k+1)=f(q(k), \psi(x(k), \hat{u}(k)), w(k)), \\
& x(k+1)=\phi(x(k), \hat{u}(k)), \\
& y(k)=h(q(k), \psi(x(k), \hat{u}(k)), w(k)) .
\end{aligned}
$$

We remark that without great difficulties one may also allow for dependency of (12) on the exogenous variables $w$; see also section 3 .

We now say that the system (1) is dynamic decouplable if there exists a model (12) such that the overall system (13) is static decouplable. For the linear model (2) we obviously require the additional model (12) to be linear too, say,

$$
\begin{aligned}
& x(k+1)=P x(k)+Q \hat{u}(k), \\
& u(k)=R x(k)+S \hat{u}(k),
\end{aligned}
$$

yielding as overall system

$$
\begin{aligned}
& q(k+1)=A q(k)+B R x(k)+B S \hat{u}(k)+E w(k), \\
& x(k+1)=P x(k)+Q \hat{u}(k), \\
& y(k)=C q(k)+D R x(k)+D S \hat{u}(k)+I w(k) .
\end{aligned}
$$

The important question now is: when is a system dynamic decouplable? Let us first review this for linear systems. To see that there are systems which are dynamic decouplable but not static decouplable consider the following mathematical model:

Example. Let

$$
\begin{aligned}
& q_{1}(k+1)=u_{1}(k), \\
& q_{2}(k+1)=q_{3}(k)+u_{1}(k), \\
& q_{3}(k+1)=u_{2}(k), \\
& y_{1}(k)=q_{1}(k), \\
& y_{2}(k)=q_{2}(k) .
\end{aligned}
$$


Then $y_{1}(k+1)=u_{1}(k)$, so $\rho_{1}=1, y_{2}(k+1)=q_{3}(k)+u_{1}(k)$, thus $\rho_{2}=1$, and the decoupling matrix is

$$
\left[\begin{array}{ll}
1 & 0 \\
1 & 0
\end{array}\right]
$$

Therefore this system is not static decouplable (Theorem 1). Now if we add to (16) the following dynamics:

$$
\begin{aligned}
& x_{1}(k+1)=x_{2}(k), \\
& x_{2}(k+1)=\hat{u}_{1}(k), \\
& u_{1}(k)=x_{1}(k), \\
& u_{2}(k)=\hat{u}_{2}(k),
\end{aligned}
$$

we see that $y_{1}(k+3)=\hat{u}_{1}(k)$ and $y_{2}(k+2)=x_{2}(k)+\hat{u}_{2}(k)$, and so $\rho_{1}=3$ and $\rho_{2}=2$, and the decoupling matrix equals

$$
\left[\begin{array}{ll}
1 & 0 \\
0 & 1
\end{array}\right]
$$

which is clearly nonsingular. So, indeed, (16) together with (17) is static decouplable.

The above Example shows that one can exploit the more general automatic policy rules (14) we allow for.

At this point it is useful to interpret a model as used in (12). It is the policy maker who has to design this model. The basic ingredient of a model like (12) is that the original instrument variables $u$, driven by the new instrument variables $\hat{u}$, come up as outputs of (12). The dynamic equation in (12) typically allows for constructing the time lags between the components of the original instrument variables. In order to achieve an economic goal as, e.g., decoupling, it might be necessary to make such a distinction between, for example, $u_{1}$ and $u_{2}$ as in the above Example; the time lag that exists before instruments appear explicitly at the target variables may increase for some components of the target variables. Simply said it takes longer hefore some target variables are effected by the instruments. This is an essential ingredient of a dynamic precompensator. The second part of dynamic precompensation is 
that of adding static control loops to the precompensated system (13) of (15). From this one can see that the dynamic control scheme includes the more usual static policy rules of, for instance, Aoki and Canzoneri (1979): simply take in (12) $x \equiv 0$ and $u(k)=\hat{u}(k)$. Notice that for decoupling purposes the introduction of a precomponsator as given here is the most general approach to the problem; cf. Wang (1970) and Wolovich (1974). In the dynamic path controllability problem - to be discussed later - the control policy scheme proposed here is just a mean to obtain the desired instruments yielding the prescribed target path.

The following theorem gives a complete description whether or not the linear system (2) is dynamic decouplable:

Theorem 2. The linear system (2) is dynamic decouplable if and only if its transfer matrix (4) has full rank.

Proof. See, e.g., Wang (1970) where an explicit procedure of designing the system (14) is given.

Note that what the system (14) is doing in the dynamic decoupling problem is in fact delaying some of the instrument variables. See, in particular, the above Example where we essentially have $u_{1}(k+2)=\hat{u}_{1}(k), u_{2}(k)=\hat{u}_{2}(k)$, i.e., we adjust the old instrument $u_{1}(\cdot)$ two time instants later by choosing $\hat{u}_{1}(\cdot)$.

An explicit algorithm for studying the dynamic decoupling problem for the nonlinear system (1) with constant exogenous variables is given in Nijmeijer (1987). This will be illustrated in the next section.

So far we have not discussed the important problem of dynamic path controllability. A definition of this property is fairly easy, namely we call the system (1) dynamic path controllable whenever there exists a fixed adjustment time $k_{0}$ [depending only on the system (1)] such that for all possible time functions $\xi(k)$ in the $m$-dimensional target space there exists a proper choice of instruments $\bar{u}(k), k \geq 0$, which yield as target variable $y(k)=\xi(k), k \geq k_{0}$. See Wohltmann (1984), Aoki and Canzoneri (1979), and other engineering references on right-invertibility. Note that generally there is a policy lead that prevents the equation $y(k)=\xi(k)$ to hold from time $k=0$ on [cf. Preston and Pagan (1982) and Wohltmann and Krömer (1984)]. This is because in most circumstances one is not free to choose the initial state $q(0)$ of the economic system (1) but has to initialize the system in a given state $q_{0}$. This implies that the equation $y(k)=\xi(k)$ holds after a certain adjustment time $k_{0}$.

There is an intimate relation between dynamic path controllability and decoupling. Let us discuss this first for the linear systems (2). If we assume 
that the corresponding linear system (7) is static decouplable (see Theorem 1 ), then there is a control law $u(k)=F x(k)+G v(k)$ which yields (10), or (11) if we take into account the effect of exogenous variables. Clearly in both cases - (10) and (11) - we may take $v_{i}(\cdot), \ldots, v_{m}(\cdot)$ arbitrary and this shows that for each $i=1, \ldots, m$ we can adjust the target variables $y_{i}\left(\rho_{i}\right)$, $y_{i}\left(\rho_{i}+1\right), \ldots$ by properly choosing $v_{1}(0), v_{i}(1), \ldots$. So in this case the delay in each channel equals $\rho_{i}$. At this point it is extremely important to emphasize the fact that one can solve 'backwards' for the desired instruments yielding the desired target variable $\xi(k)$. For if one wants $\xi(k)$ as the desired target variable one defines [see (10)]

$$
\begin{gathered}
\bar{v}_{1}(k)=\xi_{1}\left(k+\rho_{1}\right), \\
\vdots \\
\bar{v}_{m}(k)=\xi_{m}\left(k+\rho_{m}\right),
\end{gathered}
$$

and then define $\bar{u}(k)$ via (9) with $v(k)$ replaced by the $\bar{v}(k)$ defined in (18). A similar argument works for the system (2) containing exogenous variables [see (11)]. So for a static decouplable linear economic system an explicit method for dynamic path controllability is available; compare with Wohltmann and Krömer (1984b). Next we consider a linear system (2) which is dynamic decouplable; see Theorem 2. So there exists an additional model (14) such that the overall system (15) is static decouplable and so, from the foregoing reasoning, it is dynamic path controllable also. Note that, again by the use of Theorem 2, we have an explicit procedure for computing a desired instrument yielding a given target variable. In fact the result that a linear system which is dynamically decouplable is also dynamic path controllable is classical in engineering [see Brockett and Mesarovic (1965) and Sain and Massey (1969)], the only surprising fact may be that this condition is necessary and sufficient. Following Wohltmann and Krömer (1984) we summarize this as follows:

Theorem 3. The linear system (2) is dynamic path controllable if and only if the transfer matrix (4) has full rank.

Next we discuss dynamic path controllability for the general economic system (1). Let $\bar{u}(k), k=0,1,2, \ldots$, be specific instrument policy and let $\bar{y}(k)$ be the corresponding target variables [for a given initial state $q(0)=q_{0}$ ]. We call the system (1) local dynamic path controllable if there exists an $\varepsilon>0$ such that all time functions $\xi(k), k \geq k_{0}$, with $\|\xi(k)-\bar{y}(k)\|<\varepsilon$ for all $k$, are 
dynamic path controllable. So a target path that is sufficiently close to the reference path $\bar{y}(\cdot)$ can be reproduced by a suitable choice of instruments $u(\cdot)$. The following result combines the results of Theorem 2 and Theorem 3 for a general nonlinear system.

Theorem 4. The nonlinear system ( 1 ) is locally dynamic path controllable if and only if the system (1) is dynamic decouplable.

Proof. This follows along the same lines as the proof of Theorem 3.7 of Nijmeijer (1987).

The importance of the above result can be explained as follows. Suppose we are interested in the (local) dynamic path controllability of an cconomic system where the number of target variables equals the number of instrument variables. Then we first use the algorithm given in Nijmeijer (1987) to verify whether or not the system is dynamic decouplable. It is this algorithm that can directly be used for computing the desired instrument strategy which produces a prescribed target variable trajectory. An example illustrating this procedure is given in the next section.

\section{An example}

In this section we will show explicitly for a particular nonlinear economic model that it is dynamic decouplable as described in the previous section and that it therefore automatically is (local) dynamic path controllable. This example is borrowed from Wohltmann and Krömer (1984). The author is grateful to Dr. H.W. Wohltmann for his valuable comments on this example. Consider the following familiar model of a closed economy:

$$
\begin{aligned}
Y(k+1)= & Y(k)+\alpha\{C(Y(k))+I(Y(k), R(k), K(k)) \\
& \left.+P(k)^{-1} G(k)-Y(k)\right\} \\
R(k+1)= & R(k)+\beta\left\{L(Y(k), R(k))-P(k)^{-1} M(k)\right\} \\
K(k+1)= & K(k)+I(Y(k), R(k), K(k)), \\
Y(k)= & F(N(k), K(k)) \\
N(k)= & H(W(k), P(k)) .
\end{aligned}
$$


In this model the quantities have the following interpretation:

$Y=$ real output,

$C=$ real private consumption,

$I=$ real private net investment,

$R=$ nominal interest rate,

$K=$ real capital stock,

$P=$ price level,

$G=$ nominal government spending,

$L=$ real money demand,

$M=$ nominal money stock,

$N=$ labour demand,

$W=$ nominal wage rate.

In (19) and (20) $\alpha$ and $\beta$ are given positive constants. Eq. (19) is a dynamic IS equation and (20) is a dynamic LM equation. The capital accumulation is described via the dynamic Keynesian eq. (21). Eq. (22) is a macroeconomic production function and (23) defines the labour demand as a function of the real wage rate. In this model $G$ and $M$ are the instrument variables, $W$ is a known exogenous variable, and the real output $Y$ and the price level $P$ are the target variables. We first have to put (19)-(23) into the state space form (1); that is, we have to rewrite (22) and (23). We will do this around a particular steady state solution of (19)-(23), say $(\bar{Y}, \bar{R}, \bar{K}, \bar{W}, \bar{G}, \bar{M}, \bar{N})$. Note that it is not necessary to work around such an equilibrium point, but that one has to be more careful in specifying the domain where the Implicit Function Theorem applies; see Nijmeijer (1987).

Consider the relation

$$
N=H(W, P),
$$

which holds at the steady state $(\bar{N}, \bar{W}, \bar{P})$. Provided that

$$
\frac{\partial H}{\partial P}(\bar{W}, \bar{P}) \neq 0
$$

we may locally apply the Implicit Function Theorem yielding locally $P$ as a function of $N$ and $W$, say,

$$
P=\tilde{H}(W, N) \quad[\bar{P}=\tilde{H}(\bar{W}, \bar{N})]
$$


Similarly the relation

$$
Y=F(N, K),
$$

which holds true at $(\bar{Y}, \bar{N}, \bar{K})$, can locally be transformed into

$$
N=\tilde{F}(Y, K) \quad[\bar{N}=\tilde{F}(\bar{Y}, \bar{K})]
$$

provided that

$$
\frac{\partial F}{\partial N}(\bar{N}, \bar{K}) \neq 0
$$

So assuming (25) and (28) we have for the second target variable

$$
P(k)=\tilde{H}(W(k), N(k))=\tilde{H}(W(k), \tilde{F}(Y(k), K(k))) .
$$

Altogether we have obtained - locally - a model of the form (1),

$$
\begin{aligned}
& Y(k+1)=f_{1}(Y(k), R(k), K(k), W(k), G(k)), \\
& R(k+1)=f_{2}(Y(k), R(k), K(k), W(k), M(k)), \\
& K(k+1)=f_{3}(Y(k), R(k), K(k)), \\
& Q_{1}(k)=Y(k), \\
& Q_{2}(k)=P(k)=\tilde{H}(W(k), \tilde{F}(Y(k), K(k))),
\end{aligned}
$$

where $Q_{1}$ and $Q_{2}$ denote the target variables and the functions $f_{1}, f_{2}$ and $f_{3}$ directly follow from (19)-(21) and (30). Note that both target variables $Q_{1}$ and $Q_{2}$ are not directly influenced by the instrument variables $G$ and $M$. From (32) we obtain

$$
\begin{aligned}
Q_{1}(k+1)= & Y(k+1)=f_{1}(Y(k), R(k), K(k), W(k), G(k)) \\
Q_{2}(k+1)= & \tilde{H}(W(k+1), \tilde{F}(Y(k+1), K(k+1))) \\
= & \tilde{H}\left(W(k+1), \tilde{F}\left(f_{1}(Y(k), R(k), K(k), W(k), G(k)),\right.\right. \\
& \left.\left.f_{3}(Y(k), R(k), K(k))\right)\right) .
\end{aligned}
$$


We have to evaluate the dependency of (33) and (34) on the instruments $G$ and $M$. Note that both $Q_{1}(k+1)$ and $Q_{2}(k+1)$ do not explicitly depend upon $M(k)$. From the particular structure of $f_{1}$ [see (19)] we see that

$$
\frac{\partial f_{1}}{\partial G}(\bar{Y}, \bar{R}, \bar{K}, \bar{W}, \bar{G}) \neq 0 .
$$

Therefore in a neighborhood of the equilibrium point (33) explicitly depends on the instrument variable $G$. In the same way, because

$$
\frac{\partial \tilde{H}}{\partial N}(\bar{W}, \bar{N}) \cdot \frac{\partial \tilde{F}}{\partial Y}(\bar{Y}, \bar{K}) \neq 0
$$

[this follows from (25)-(28)], also (34) explicitly depends on the instrument $G$. Define the 'inverse' control policy

$$
\tilde{G}=f_{1}(Y, R, K, W, G) \text {. }
$$

This 'inverse' control law (37) is nothing else but a (linear) convex combination of the real output $Y$ and the aggregate demand $D:=C(Y)+I(Y, R, K)$ $+P^{-1} G$; see the defining cq. (19) for $f_{1}$. In the special case $\alpha=1$, which corresponds with a Lundberg lag of one period between the aggregate supply and demand, $\hat{G}$ equals the aggregate demand $D$. By itself, (37) defines $\hat{G}$ as an endogenous variable and one could give it the interpretation of an (artificial) intermediate target variable. Transforming (37) via the Implicit Function Theorem around the equilibrium point [see (35)], we obtain

$$
G=f_{1}(Y, R, K, W, \tilde{G}),
$$

in which $\tilde{G}$ has to be considered as a (new) exogenous instrument variable. In the special case $\alpha=1$, this amounts to taking $G=P \cdot D+P \cdot C(Y)$ $-P \cdot I(Y, R, K)$ and interpreting the aggregate demand $D$ as the instrument variable. Note that this is precisely an equation of the form (11) [see also Aoki (1974) where such generating of instrument variables is used].

Plugging (38) in (33) and (34) yields

$$
\begin{aligned}
& Q_{1}(k+1)=\tilde{G}(k), \\
& Q_{2}(k+1)=\tilde{H}\left(W(k+1), \tilde{F}\left(\tilde{G}(k), f_{3}(Y(k), R(k), K(k))\right)\right) .
\end{aligned}
$$

At this point we may conclude [see, e.g., Nijmeijer (1987)] that this system is 
not static decouplable. This is because in both eqs. (39) and (40) only the instrument $\tilde{G}$ is present. This shows that even when we take a linear model of a closed economy - so (19)-(23) are linear equations - then this system is not static feedback decouplable. On the other hand such a linear model, or as we will see later its linearization [cf. Wohltmann and Krömer (1984)], is in fact dynamic path controllable under one additional constraint. Indeed it satisfies one of the conditions given in Aoki and Canzoneri (1979, p. 607, cond. C), but it fails to be static decouplable. This shows that the results on static decoupling of Aoki and Canzoneri (1979) are not completely true.

Next we introduce a one-step delay for the 'instrument' $\tilde{G}$. So define

$$
\begin{aligned}
& Z(k+1)=\hat{G}(k), \\
& \tilde{G}(k)=Z(k) .
\end{aligned}
$$

The system (31) together with (41) becomes

$$
\begin{aligned}
& Y(k+1)=Z(k), \\
& Z(k+1)=\hat{G}(k), \\
& R(k+1)=f_{2}(Y(k), R(k), K(k), W(k), M(k)), \\
& K(k+1)=f_{3}(Y(k), R(k), K(k)) .
\end{aligned}
$$

Notice that, in particular when there is a Lundberg lag of one period between aggregate supply and demand, i.e., $\alpha=1$, (41) requires the new instrument $\hat{G}$ which in fact equals aggregate demand one time instant earlier: $\hat{G}(k)=$ $D(k-1)$.

Next we compute $Q_{1}(k+2)$ and $Q_{2}(k+2)$ :

$$
\begin{aligned}
Q_{1}(k+2)= & \hat{G}(k), \\
Q_{2}(k+2)= & \tilde{H}(W(k+2), \tilde{F}(\tilde{G}(k+1), \\
& \left.\left.f_{3}(Y(k+1), R(k+1), K(k+1))\right)\right) \\
= & \tilde{H}\left(W(k+2), \tilde{F}\left(\hat{G}(k), f_{3}(Z(k),\right.\right. \\
& f_{2}(Y(k), R(k), K(k), W(k), M(k)), \\
& \left.\left.\left.f_{3}(Y(k), R(k), K(k))\right)\right)\right) .
\end{aligned}
$$


Note that $Q_{1}(k+2)$ directly depends on the new instrument $\hat{G}(k)$ and not on $M(k)$. Moreover $Q_{2}(k+2)$ is - in a neighborhood of the equilibrium point - influenced by $M(k)$ if

$$
\begin{aligned}
& \frac{\partial \tilde{H}}{\partial N}(\bar{W}, \bar{N}) \cdot \frac{\partial \tilde{F}}{\partial K}(\tilde{Y}, \bar{K}) \\
& \cdot \frac{\partial f_{3}}{\partial R}(\bar{Y}, \bar{R}, \bar{K}) \cdot \frac{\partial f_{2}}{\partial M}(\bar{Y}, \bar{R}, \bar{K}, \bar{W}, \bar{M}) \neq 0 .
\end{aligned}
$$

From the particular structure of the function $f_{2}$ [see (31) and (20)] it follows that

$$
\frac{\partial f_{2}}{\partial M}(\bar{Y}, \bar{R}, \bar{K}, \bar{W}, \bar{M}) \neq 0,
$$

and so, using (36), we see that

$$
\frac{\partial \tilde{F}}{\partial K}(\bar{Y}, \bar{K}) \cdot \frac{\partial f_{3}}{\partial R}(Y, R, K) \neq 0
$$

must hold for (45) to be true. See Wohltmann and Krömer (1984) for an economic interpretation of (47). So, provided (45) holds, we can define the 'inverse' control law

$$
\hat{M}=\tilde{H}\left(W_{1}, \tilde{F}\left(\hat{G}, f_{3}\left(Z, f_{2}\left(Y, R, K, W_{2}, M\right), f_{2}(Y, R, K)\right)\right)\right),
$$

which by the Implicit Function Theorem is locally equivalent to the control policy

$$
M=\xi\left(W_{1}, \hat{G}, Z, Y, R, K, W_{2}, \hat{M}\right)
$$

We remark that in the control policy (49) [see also (48)] the monetary policy instrument $M$ depends on both new instruments $\hat{M}$ and $\hat{G}$. So it is not possible to make a one-to-one linking between the old instrument $M$ and the new instrument $\hat{M}$, like it was the case for $G$ and $\hat{G}$. In fact, one can even show that in a situation where static decoupling is impossible one always will have such a coupling of instruments. We refer to Aoki and Canzoneri (1979) for a discussion of this point for static decouplability; for linear systems the essential conditions is that the matrix $M$ appearing in (8) is diagonal and nonsingular. 
Notice also that a similar discussion as has been given after eq. (37), relating the variables $G$ and $\hat{G}$, could be given about the character of the variables $M$ and $\hat{M}$ of eq. (48) in relation to (49). We leave that for the reader.

Plugging (48) in into (43) and (44) yields

$$
\begin{aligned}
& Q_{1}(k+2)=\hat{G}(k), \\
& Q_{2}(k+2)=\hat{M}(k),
\end{aligned}
$$

i.e., via the control policy defined in (37), (38), (41), (48) and (49) the overall system is decoupled! Note that the new instruments $\hat{G}$ and $\hat{M}$ in general have to be bounded to a certain region; this follows from the Implicit Function Theorem, which has been used to define these controls. Obviously [see Theorem 4] the closed economy is locally dynamic path controllable. So indeed, in a local sense, from eq. (50) it follows that the new instruments $\hat{G}$ and $\hat{M}$, respectively, can be used to steer the real output $Y$ and the price level target $P$, respectively. In effect only the original $G$ is needed to steer $Y$, and the instrument $M$ depending on the already chosen instrument $G$ will be used in controlling $P$. In order to find the desired strategy yielding the prescribed targets $\left[\bar{Q}_{1}(k), \bar{Q}_{2}(k)\right]$ use (50) and solve backwards using the eqs. (49), (41) and (38). We remark that this result is in agreement with the result of Wohltmann and Krömer (1984), where the dynamic path controllability has been proved for the linearization of the model (19)-(23) under the condition (47) [see Wohltmann and Krömer (1984, p. 325)]. So what is shown to be true for the linearization of the model is also valid for the model itself. To achieve the dynamic path controllability one of course needs to work with the model itself and, therefore, one in principle needs to use the nonlinear policy scheme described here. What is reassuring here is of course that the result for the linearized model is in agreement with the nonlinear result. Let us finally emphasize that our control strategy proposed here - based on an iterative use of the Implicit Function Theorem - is potentially quite well solvable for given functions appearing in (19)-(23); see, e.g., Schwetlick (1979).

\section{Concluding remarks}

One of the important questions a policy maker asks himself is whether or not he is able to steer the target variables in an economic model along a desired time path using only the instrument variables. So far in the economic literature this problem has only been addressed for linear models. However, most models used in economics are typically nonlinear. Therefore a usual approach for answering this question is based on linearization of the nonlinear model. Hopefully the conclusion one obtains when working with the linearized model is also true for the nonlinear economic system too. For a particular 
example - a closed economy - we have shown that this conclusion is indeed true. The method for dynamic path controllability in this paper is based on the observation that dynamic path controllability and dynamic decouplability of a system are equivalent properties. In this way an explicit way of solving the dynamic path controllability is by checking if the economic model is dynamic decouplable. In this context dynamic decoupling is a fairly general scheme of well-defined policy rules which guarantee that each of the (new) instrument variables only influences one target variable. Obviously the dynamics of the policy rules we use depend crucially upon the structure of the economic model postulated. But this is of course fully in agreement with our expectations.

\section{References}

Albrecht, F., K.A. Grasse and N. Wax, 1981, Reproducibility of linear and nonlinear input-output systems, Journal of Mathematical Analysis and Applications 79, 178-202.

Aoki, M., 1974, Non-interacting control of macroeconomic variables, implications on policy mix considerations, Journal of Econometrics 2, 261-281.

Aoki, M., 1975, On a generalization of Tinbergen's condition in the theory of policy to dynamic models, Review of Economic Studies 42, 293-296.

Aoki, M., 1976, Optimal control and system theory in dynamic economic analysis (NorthHolland, Amsterdam).

Aoki, M. and M. Canzoneri, 1979, Sufficient conditions for control of target variables and assignment of instruments in dynamic macroeconomic models, International Economic Review 20, 605-615.

Brockett, R.W. and M.D. Mesarovic, 1965, The reproducibility of multivariable systems, Journal of Mathematical Analysis and Applications 11, 548-563.

Buiter, W.H., 1979, Unemployment-inflation trade-offs with rational expectations in an open economy, Journal of Economic Dynamics and Control 1, 117-141.

Claude, D., 1983, Découplage des systèmes: Du linéaire au nonlinéaire, in: I.D. Landau, ed., Dévelopments et utilisations d'outils et modèles mathematique en automatique, analyse de systèmes et traitement du signal, Vol. 3 (C.N.R.S., Paris) 533-555.

Descusse, J. and C. Moog, 1985, Decoupling with dynamic compensation for strong invertible affine nonlinear systems, International Journal of Control 42, 1387-1398.

Falb, P.L. and W.A. Wolovich, 1967, Decoupling in the design and synthesis of multivariable control systems, IEEE Transactions on Automatic Control AC-12, 651-659.

Isidori, A., 1985, Nonlinear control systems: An introduction, Lecture notes in control and information-sciences 72 (Springer-Verlag, Berlin).

Lovass-Nagy, V., R.J. Miller and D.L. Powers, 1976, On output control in the servomechanism sense, International Journal of Control 24, 435-440.

Lovass-Nagy, V., R.J. Miller and D.L. Powers, 1978, Further results on output control in the servomechanism sense, International Journal of Control 27, 133-138.

Maciejowski, J.M. and D. Vines, 1984, Decoupled control of a macroeconomic model using frequency-domain methods, Journal of Economic Dynamics and Control 7, 55-77.

Nijmeijer, H., 1986, On the input-output decoupling of nonlinear systems, in: $M$. Fliess and $M$. Hazewinkel, eds., Algebraic and geometric methods in nonlinear control theory (Reidel, Dordrecht) 101-119.

Nijmeijer, H., 1987, Local (dynamic) input-output decoupling of discrete time nonlinear systems, IMA Journal of Mathematical Control and Information 4, 237-250.

Nijmeijer, H. and W. Respondek, 1986, Decoupling via dynamic compensation for nonlinear control systems, in: IEEE proceedings of the 25 th conference on decision and control (Athens) $192-197$. 
Preston, A.J. and A.R. Pagan, 1982, The theory of economic policy (Cambridge University Press, Cambridge).

Sain, M.K. and J.L. Massey, 1969, Invertibility of linear time-invariant dynamical systems, IEEE Transactions on Automatic Control AC-14, 141-149.

Schwetlich, H., 1979, Numerische Lösung nichtlinearer Gleichungen (Oldenbourg Verlag, München)

Sinha, P.K., 1977, State feedback decoupling of nonlinear systems, IEEE Transactions on Automatic Control AC-22, 487-489.

Wang, S.H., 1970, Design of precompensator for decoupling problems, Electronics Letters 6, $739-741$.

Wohltmann, H.-W., 1984, A note on Aoki's condition for path controllability of continuous-time dynamic economic systems, Review of Economic Studies 51, 343-349.

Wohltmann, H.-W., 1985a, Function space dependent criteria for target path controllability of dynamical systems, International Journal of Control 41, 709-715.

Wohltmann, H.-W., 1985b, On the controllability of continuous-time macroeconomic models, Journal of Economics 45, 47-66.

Wohltmann, H.-W. and W. Krömer, 1983, A note on Buiter's sufficient condition for perfect output controllability of a rational expectations model, Journal of Economic Dynamics and Control 6, 201-205.

Wohltmann, H.-W. and W. Krömer, 1984, Sufficient conditions for dynamic path controllability of economic systems, Journal of Economic Dynamics and Control 7, 315-330.

Wolovich, W.A., 1974, Linear multivariable systems (Springer-Verlag, Berlin). 